\title{
STEREO: Search for sterile neutrinos at the ILL
}

\author{
Luis Manzanillas* ${ }^{\dagger}$ \\ LAPP - IN2P3 - CNRS / Université Savoie Mont Blanc - Université Grenoble Alpes \\ E-mail: lmanzanillas@gmail.com
}

\begin{abstract}
Recent studies have shown that there are discrepancies between observations and the theoretical predictions in some neutrino experiments at short distances. In the so-called "Reactor Antineutrino Anomaly" and in the "Gallium Anomaly", these differences from the expectations are at the $\sim 3 \sigma$ level in both cases. Oscillations into a light sterile neutrino state $\left(\Delta m^{2} \sim 1 e V^{2}\right)$ could account for the deficits in observed rates. The STEREO experiment has been conceived to confirm or reject the sterile neutrino hypothesis. It will search for an oscillation pattern at short baselines (9-11 m) in the energy spectrum of the antineutrinos emitted by the research nuclear reactor of the Institut Laue Langevin. To this end, the detector is filled with two tons of Gd-loaded liquid scintillator read out by an array of PMTs and is segmented into 6 cells in the direction of the antineutrino's propagation. STEREO should be capable of excluding the best fit parameters region of a candidate sterile neutrino at $5 \sigma$. Data taking will start at the end of 2016 and the first physics results are expected by the first semester of 2017.
\end{abstract}

Neutrino Oscillation Workshop

4 - 11 September, 2016

Otranto (Lecce, Italy)

* Speaker.

${ }^{\dagger}$ On behalf of the STEREO collaboration 


\section{Introduction}

During the preparation of the current $v$ experiments devoted to the measurement of $\theta_{13}$ (Double Chooz, Daya Bay and RENO), the $v$ spectra and fluxes of nuclear reactors were reevaluated. The new estimations increased the predicted antineutrino flux by about $3 \%[1,2]$. Using the new estimations, a reevaluation of previous experiments' observed antineutrino flux, a total deficit of around $7 \%(3 \sigma)$ was found [3]. This is known as the reactor antineutrino anomaly (RAA). Oscillations into a light sterile $v$ could account for such deficits [4]. In addition, other anomalies pointing to the sterile $v$ hypothesis have also been observed in other experiments, which are known as the Gallium and LSND anomalies [5].

In the $3+1 v$ framework, the oscillation probability induced by a sterile $v$ state at the $\mathrm{eV}^{2}$ scale can be reduced to the two flavor approximation. Thus, the survival probability is given by [6]

$$
P_{\overline{v_{e}} \rightarrow \overline{v_{e}}}^{3+1} \simeq 1-\sin ^{2} 2 \theta_{e e} \sin ^{2}\left(\frac{\Delta m_{41}^{2} L}{4 E_{V}}\right)
$$

where $\theta$ and $\Delta m^{2}$ are respectively the mixing angle between the two flavors and the difference of squared masses $m_{1}^{2}-m_{4}^{2}$, both being parameters given by Nature. $E_{v}$ is the $v$ energy and $L$ is the distance traveled by the $v$ from the source to the detection point. In most of the reactor experiments, the $\overline{v_{e}}$ energy spectrum is measured at a fixed distance. However, since the uncertainties in the prediction of this spectrum are high, searching for a new oscillation requires an unambiguous signature: the distortion of this energy spectrum as function of the distance. The best fit values of $\theta$ and $\Delta m^{2}$ suggested by the reactor and gallium anomalies [3] $\left(\Delta m^{2} \simeq 2.3 e V^{2}, \sin ^{2} 2 \theta \simeq 0.14\right)$ implies an oscillation of about $3 \mathrm{~m}$ for $\overline{v_{e}}$, $\mathrm{s}$ of $3 \mathrm{MeV}$, which is a typical energy of detected reactor $\overline{v_{e}}$ 's. Thus, the other key ingredient to search for such oscillations is to have a compact source.

A broad experimental program is ongoing to test the sterile $v$ hypothesis using different detection techniques and $v$ sources. Among the experiments using a nuclear reactor as $v$ source we have the STEREO experiment, whose goal is to confirm or reject the existence of a light sterile $v$ state, by searching for an oscillation pattern at short baselines in the reactor $\overline{v_{e}}$ energy spectrum. The source of $\overline{v_{e}}$ 's for the STEREO experiment is the most compact (37 cm diameter) research nuclear reactor in France, located at the Laue-Langevin Institute (ILL) in Grenoble. This reactor uses highly enriched ${ }^{235} \mathrm{U}$, which reduces the contribution from other fissile isotopes whose $\overline{v_{e}}$ spectrum is less well known than ${ }^{235} \mathrm{U}$ 's. The center of STEREO is placed $10 \mathrm{~m}$ away from the reactor core.

\section{The STEREO experiment}

STEREO will exploit a proven $\overline{v_{e}}$ detection technology, using 2 tons of Gd-loaded liquid scintillator (LS). The detector is segmented into 6 cells $\left(40 \times 90 \times 90 \mathrm{~cm}^{3}\right)$ in the direction of the $\overline{v_{e}}$ 's propagation (see figure 1). Each cell is read out by an array of 4 PMTs on the top. The target cells are surrounded by an outer crown of $30 \mathrm{~cm}$ thickness called gamma catcher (GC), which is filled with LS without Gd. The main function of the GC is to improve the energy resolution by recovering some of the escaping gammas from the target cells. At the same time, the GC can be used as veto to remove backgrounds coming from the outside. In order to further protect the detector from fast and thermal neutrons and gammas, a passive shielding of polyethylene, lead and boron loaded rubber 
encloses the entire detector. Finally, to remove background events induced by cosmic muons, a water Cerenkov detector called "muon veto" (MV) is placed on top of the detector.
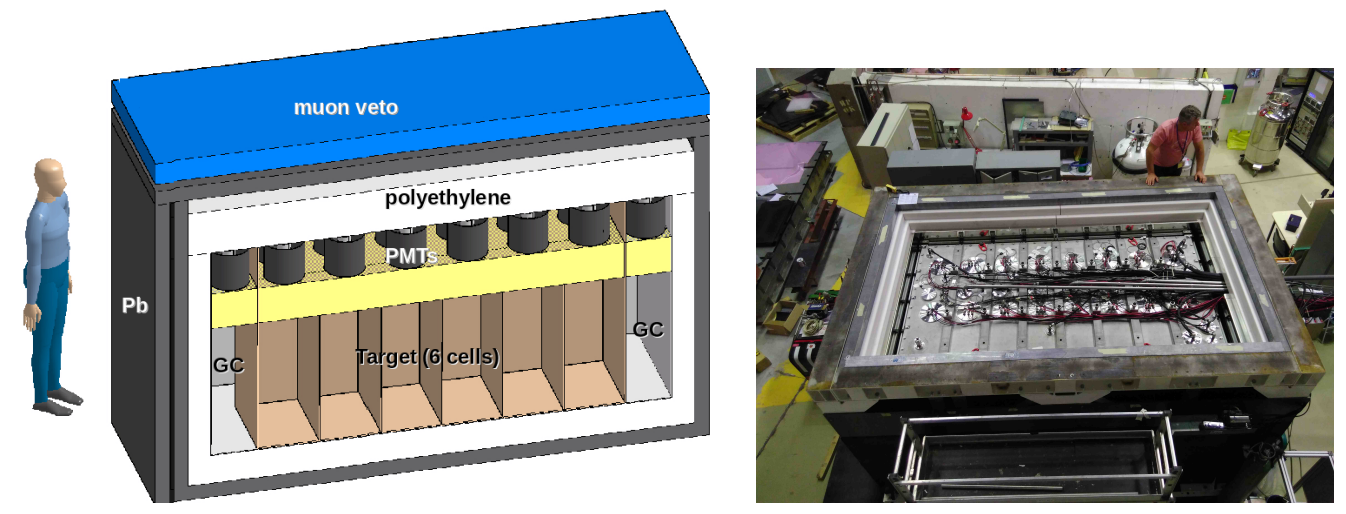

Figure 1: Left: Sketch of the different parts of the STEREO experiment, which consists of a target of 6 cells filled with LS doped with gadolinium, surrounded by an outer crown of LS called gamma catcher (GC). The scintillation light is detected by PMTs on top of the target cells and the GC. Shielding is composed of polyethylene and lead. A muon detector is placed on the top. Right: Installation of STEREO at the ILL

The $\overline{v_{e}}$ 's are detected via the inverse beta decay (IBD) process: $\overline{v_{e}}+p \rightarrow e^{+}+n$, which produces two signals correlated in time and distance. The positron deposits almost instantaneously its energy in the LS, which, together with the deposited energy of the two $511 \mathrm{keV}$ gammas from its annihilation with an electron, gives a prompt signal. The neutron thermalizes and then diffuses until it's captured by a nucleus of Gd some $\sim 15 \mu$ s later, giving rise to a delayed signal, which consists of a gamma cascade with a total energy of $8 \mathrm{MeV}$ produced by the de-excitation of the $\mathrm{Gd}$.

\section{Status of the experiment and expected physics results}

A dedicated electronics hosted in a $\mu$ TCA crate was developed and tested using the MV in 2015 [7]. In Spring of 2016 the inner detector (Target and GC) was assembled. During the summer the supporting structure and all the large items required for the STEREO shielding were delivered and mounted at the ILL. In August the inner detector was inserted inside the shielding structure and the calibration system installed. Finally, the shielding roof was closed and the MV was installed at the beginning of September. The detector will be moved to the STEREO site by the end of September and will be filled with LS at the beginning of November. Commissioning and $v$ data taking are expected to start around the beginning of November with the new reactor cycle.

The expected exclusion contour of STEREO for 300 days of data taking is shown in figure 2. The main assumptions are: a signal to background ratio of 1.5, an uncertainty in the energy scale lower than $2 \%$, an energy resolution of $10 \%$ at $2 \mathrm{MeV}$, and an energy threshold for the prompt and delayed signals of 2 and $5 \mathrm{MeV}$ respectively. Most of the region of the parameter space allowed by the RAA will be covered by STEREO. In addition, with around $400 v$ events per day, STEREO will provide a new spectrum of reference for ${ }^{235} \mathrm{U}$ with more than $100000 v$ 's. This new spectrum could provide information to understand the origin of the bump around $5 \mathrm{MeV}$ observed in the reactor $\overline{V_{e}}$ spectrum in current reactor $v$ experiments [8]. Before the spring shutdown of 2017, around 100 days of data taking are expected, enough to cover the best fit region of the RAA. 


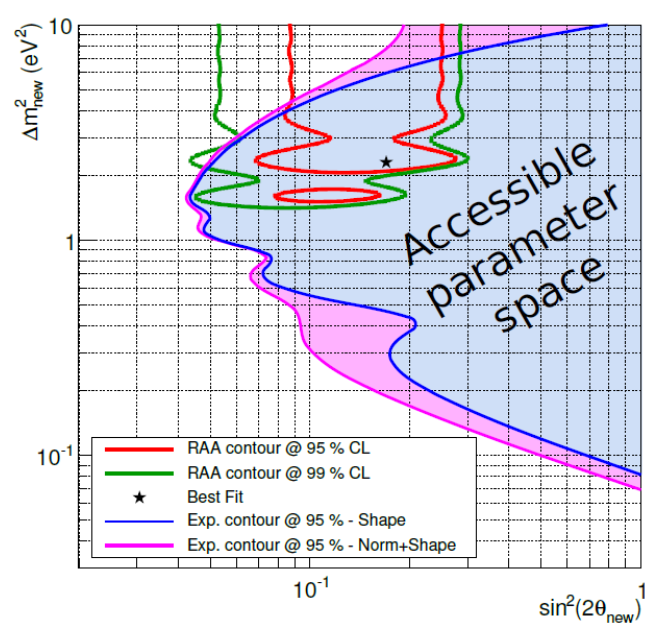

Figure 2: Expected exclusion contour of STEREO for 300 days of data taking

\section{Conclusions}

Most of the large items of STEREO have been delivered and installed at the ILL. The installation will be completed by October of 2016. The data taking will start by the beginning of November. The first physics results are expected by the first half of 2017 with around 100 days of data that can be accumulated before the spring shutdown of 2017. Thus, STEREO will provide the first search for sterile $v$ 's with an analysis of the reactor energy spectrum as function of the distance.

\section{References}

[1] T. A. Mueller et al., Improved Predictions of Reactor Antineutrino Spectra, Phys. Rev. C 83, 054615 (2011) [arXiv:1101.2663].

[2] P. Huber, On the determination of anti-neutrino spectra from nuclear reactors, Phys. Rev. C 84, 024617 (2011) [arXiv:1106.0687].

[3] G. Mention, M. Fechner, T. Lasserre, T. A. Mueller, D. Lhuillier, M. Cribier and A. Letourneau, The Reactor Antineutrino Anomaly, Phys. Rev. D 83, 073006 (2011) [arXiv:1101.2755].

[4] K. N. Abazajian et al., Light Sterile Neutrinos: A White Paper, [arXiv:1204.5379].

[5] C. Giunti and M. Laveder, Short-Baseline Active-Sterile Neutrino Oscillations?, Mod. Phys. Lett. A 22, 2499 (2007) [hep-ph/0610352].

[6] C. Giunti and C. W. Kim, Fundamentals of Neutrino Physics and Astrophysics, Univ. Pr., Oxford 2007

[7] O. Bourrion et al., Trigger and readout electronics for the STEREO experiment, JINST 11, C02078 (2016) [arXiv:1510.08238].

[8] C. Buck, A. P. Collin, J. Haser and M. Lindner, Investigating the Spectral Anomaly with Different Reactor Antineutrino Experiments, [arXiv:1512.06656]. 